\title{
The effect of rumen adaptation to oxalic acid on selection of oxalic-acid-rich plants by goats
}

\author{
Alan J. Duncan*, Pilar Frutos $†$ and Sheila A. Young \\ Macaulay Land Use Research Institute, Craigiebuckler, Aberdeen AB15 8QH, UK
}

(Received 12 November 1998 - Revised 5 May 1999 - Accepted 16 July 1999)

\begin{abstract}
Rumen microbial degradation is an important route for detoxification of secondary plant compounds encountered in the diets of free-grazing ruminants. Exposure to diets containing particular secondary plant compounds can lead to increased rates of secondary compound degradation in the rumen. An experiment was conducted to determine whether rumen adaptation to oxalic acid would influence the diet selection of goats offered choices between plant species differing in their oxalic acid content. Twelve adult female goats were divided into two groups of six animals each. One group received a daily oral dose, in gelatin capsules, of $0.6 \mathrm{mmol}$ oxalic acid/kg live weight per $\mathrm{d}$ throughout the experiment while the other group received placebos consisting of empty gelatin capsules. After an adaptation period of $8 \mathrm{~d}$, the animals were allowed to graze a mixture of spinach (rich in oxalic acid) and cabbage (low in oxalic acid) for $7 \mathrm{~h} / \mathrm{d}$ on two consecutive days per week during four consecutive 1-week periods. Intervening days were spent on grass pasture. Diet composition and intake were measured using cuticular wax $n$-alkanes as internal markers. Results showed that adapted goats included a higher proportion of spinach in their diet $(P<0.05)$ although absolute intakes of spinach were the same for the two groups. Goats in the oxalic-acid-adapted group consumed less cabbage than control animals $(P<0.05)$ suggesting that adaptation to oxalic acid at the rumen level may have interfered with detoxification of cabbage-derived secondary plant compounds. Voluntary intake increased progressively through the four experimental periods $(P<0 \cdot 001)$ with a tendency for higher intakes among control than among adapted animals $(P<0 \cdot 1)$. The experiment demonstrates how differences in the rate of degradation of secondary plant compounds may influence diet selection in ruminants.
\end{abstract}

\section{Diet selection: Oxalic acid: Rumen detoxification}

Certain plant secondary compounds have toxic properties and have been found to deter herbivore feeding on particular plant species in a large number of contexts (Palo \& Robbins, 1991). This phenomenon has an important influence on the diet selected by herbivores and therefore affects the impact of foraging animals on vegetation composition over time (Jefferies et al. 1994). One of the mechanisms whereby herbivores avoid potentially toxic food items may be through conditioned food aversions (Provenza \& Balph, 1987). Thus herbivores may learn to associate plant cues with toxic post-ingestive consequences over a series of encounters and show partial aversion to toxic plants during subsequent encounters. The strength of aversion to toxic sources is known to depend on the strength of postingestive physiological effects (du Toit et al. 1991). This behavioural adaptation to the presence of secondary plant compounds in the diet has been shown to operate in certain simple situations using artificial feeding deterrents and penfed domestic ruminants (Provenza et al. 1994). The significance of conditioned food aversions as mediators of diet selection by free-ranging herbivores is still in question.

As well as behavioural mechanisms for limiting the toxicity of plant secondary compounds, large herbivores are equipped with important physiological detoxification mechanisms (Smith, 1992). Detoxification of ingested plant secondary compounds has been shown to operate both in the digestive tract and at the systemic level. The flexibility of these detoxification mechanisms gives large herbivores the potential to adapt readily to inclusion of new toxic items in their diet, provided sufficient time is available for induction of detoxification pathways. For example, oxalic acid is present in a range of food plants commonly consumed by herbivores and renal toxicity and hypocalcaemia may occur after abrupt exposure to oxalic-acid-containing plants

\footnotetext{
Abbreviations: MTBSTFA, $N$-(tert-butyldimethylsilyl)- $N$-methyltrifluoroacetamide.

* Corresponding author: Dr Alan Duncan, fax +44 (0) 1224 311556, email a.duncan@mluri.sari.ac.uk

$\dagger$ Present address: Estación Agrícola Experimental, Consejo Superior de Investigaciones Científicas (CSIC), Apdo 788. 24080-León, Spain.
} 
(James, 1972). Gradual exposure to increasing levels of oxalic acid leads to a change in the composition of the rumen microbial population and the resulting breakdown of oxalic acid prevents these symptoms (Allison et al. 1977; Duncan et al. 1997).

Different rates of secondary plant compound detoxification in the digestive tract, arising as a result of adaptation, are likely to yield different rates of absorption of secondary compounds and therefore differences in post-absorptive consequences. An experiment is reported which tested the impact of rumen adaptation to a secondary plant compound on learned aversion to a potentially toxic plant. Goats were adapted to oxalic acid by regular oral administration; they were then offered choices between plants containing high and low concentrations of oxalic acid and their diet selection was compared with that of non-adapted animals.

\section{Materials and methods}

\section{Experimental design}

Twelve female Scottish Cashmere goats (mean live weight $38 \cdot 3$ (SD 3.47) kg) were assigned to two treatment groups of six animals each, balanced for live weight. One group was adapted to oxalic acid by once daily oral dosing $(0.6 \mathrm{mmol} /$ $\mathrm{kg}$ live weight per $\mathrm{d}$ ) throughout the experiment (treatment group) while the other group received a placebo (control group). The experiment consisted of a preliminary $8 \mathrm{~d}$ adaptation period followed by four consecutive experimental periods lasting $7 \mathrm{~d}$ each. In total, there were eight $5 \times$ $10 \mathrm{~m}$ spinach-cabbage plots and during each spinachcabbage grazing day, goats were given access to a fresh plot of spinach (Spinacea oleracea; high concentrations of oxalic acid) and cabbage (Brassica oleracea; low concentrations of oxalic acid) plants on the first $2 \mathrm{~d}$ of each experimental period. DM intake and diet composition were measured during this time. During the remaining $5 \mathrm{~d}$ of each period, goats were allowed to graze a perennial ryegrass pasture.

\section{Procedures}

Oxalic acid (Aldrich, Poole, Dorset, UK) contained in gelatin capsules (Davcaps, Monmouth, Gwent, UK) was administered to animals in the treatment group by once daily oral dosing at 09.00 hours. Placebos in the form of empty gelatin capsules were given at the same time to the control group of goats. During the preliminary $8 \mathrm{~d}$ adaptation period, oxalic acid dose rates were increased from zero to the full dose in equal increments over the first $5 \mathrm{~d}$ and the full dose was given for the remaining $3 \mathrm{~d}$. During each of the subsequent experimental periods oxalic acid was administered daily at the full dose rate except on days during which animals had access to the spinach-cabbage plots. On these days, no oxalic acid was administered to avoid potential toxicity from the combined effects of naturally ingested and artificially administered oxalic acid. Rumen samples were collected under anaerobic conditions by stomach tube at 10.00 hours on the day before, and the day after, the spinach-cabbage feeding days. Animals were taken indoors for this purpose. During each experimental period, spot samples of faeces were collected by rectal grab sampling immediately before releasing animals onto a spinachcabbage plot (09.00 hours) and at 24, 33, 48, 55 and $72 \mathrm{~h}$ thereafter. Faecal samples were stored at $-20^{\circ}$ pending $n$-alkane analysis for estimation of herbage intake and diet composition.

\section{Diet options}

From 09.00 hours to 16.00 hours on each of the days when spinach and cabbage were offered, goats had access to a fresh experimental plot measuring $5 \times 10 \mathrm{~m}$ containing cabbage (variety 'First Early Market 218'; Breeders Seeds, Ormskirk, Lancs., UK) and spinach (variety 'Carambole'; Breeders Seeds) plants sown 10 weeks before the start of the first experimental period. Each plot contained alternate spinach and cabbage plants at $0.3 \mathrm{~m}$ intervals. A fresh $50 \mathrm{~m}^{2}$ plot was used on each spinach-cabbage feeding day. Animals grazed a perennial ryegrass pasture overnight between the two spinach-cabbage feeding days and during the remaining $5 \mathrm{~d}$ of each experimental period.

\section{Measurements}

Rates of oxalic acid degradation were measured by a modification of the method of Allison et al. (1985) as described by Duncan et al. (1997). Samples of ruminal contents obtained by stomach tube were collected, kept at $37^{\circ}$ and processed within $60 \mathrm{~min}$ of collection.

Standing biomass of DM was measured by cutting, to ground level, six randomly chosen plants of each species per feeding period, pooling herbage by species and drying at $85^{\circ}$. The mean dry weight per plant was multiplied by the number of plants counted per plot to determine total biomass of DM per hectare. A further three randomly chosen plants of each species per period were cut to ground level, pooled by species and subsequently subsampled for oxalic acid analysis. The remaining material was then freeze-dried for further chemical analysis. Samples were then milled through a $1 \mathrm{~mm}$ screen and analysed for total N (Perkin Elmer elemental analyser; Perkin Elmer Ltd, Beaconsfield, Bucks., UK), neutral-detergent fibre (Van Soest \& Wine, 1967), acid-detergent fibre (Van Soest, 1963) and lignin (Van Soest, 1963). Samples were analysed for oxalic acid by GC. Fresh, frozen samples were prepared by a modification of the method of Libert (1981) as follows. Fresh herbage $(20 \mathrm{~g})$ was homogenized in $250 \mathrm{ml}$ water for $3 \mathrm{~min}$. To $25 \mathrm{ml}$ homogenate, $20 \mathrm{ml} 5 \mathrm{M}-\mathrm{HCl}$ was added along with $0.5 \mathrm{ml}$ internal standard $(2 \mathrm{mg}$ succinic $\mathrm{acid} / \mathrm{ml}$ absolute alcohol) and distilled water to make up to $50 \mathrm{ml}$. The homogenate was heated in a water-bath at $95^{\circ}$ for $30 \mathrm{~min}$ and then filtered. Oxalic acid was measured using GC following preparation of derivatives by silylation with $N$-(tert-butyldimethylsilyl)- $N$-methyltrifluoroacetamide (MTBSTFA; Aldrich). For this purpose homogenates were acidified and extracted with diethyl ether. Ether was removed by evaporation and MTBSTFA reagent $(100 \mathrm{ml} / \mathrm{l}$ solution in acetonitrile) was added before heating at $80^{\circ}$ for $30 \mathrm{~min}$. Samples were analysed by capillary GC using a 
Supelco SBP-20 bonded phase capillary column $(0.53 \mu \mathrm{m} \times$ $30 \mathrm{~m}$ ) with oven temperature programmed from $60^{\circ}$ to $200^{\circ}$ at $7 \% \mathrm{~min}$.

Herbage intake and diet composition during spinachcabbage feeding periods were estimated using a modification of the $n$-alkane technique (Dove \& Mayes, 1991) modified for estimation of short-term intake and diet composition (Duncan et al. 1999). Briefly, the method relies on the use of $n$-alkanes which are components of the cuticular wax of most plants. $n$-Alkanes are relatively indigestible and can thus be used as internal markers for intake estimation following collection and analysis of faecal samples. The accuracy of estimation is markedly improved by oral administration of synthetic $n$-alkanes with similar chain lengths, which allows correction for incomplete faecal recovery of herbage $n$-alkanes. Diet composition can be estimated from faecal $n$-alkane patterns by virtue of the differing $n$-alkane profiles of different plant species. In the current experiment, for example, the fact that cabbage contains high levels of $\mathrm{C}_{29} n$-alkane, while spinach is rich in $\mathrm{C}_{31} n$-alkane, was exploited. Measurement of faecal concentrations of these two $n$-alkanes allowed accurate estimation of the proportions of the two source plant components in the diet. In the present experiment, animals were dosed with two $n$-alkane pellets on each dosing occasion at $0,7,24$ and $31 \mathrm{~h}$ after the start of each $2 \mathrm{~d}$ spinach-cabbage feeding period. Each $n$-alkane pellet consisted of a paper bung impregnated with $200 \mathrm{mg} \mathrm{C}_{28} n$-alkane and $200 \mathrm{mg}$ of $\mathrm{C}_{32}$ $n$-alkane. Excretion curves of dosed and herbage $n$-alkanes were plotted using $n$-alkane concentrations measured in faecal grab samples. The areas under the excretion curves for each $n$-alkane were used in place of true $n$-alkane concentrations to estimate intake and diet composition according to conventional $n$-alkane calculation methods (Dove \& Mayes, 1991). Intake of grass during the rest period between each cabbage-spinach grazing day was not calculated. The contribution of $n$-alkanes derived from grass consumed during this time to the excretion curves was disregarded since $n$-alkane concentrations in Lolium perenne (100-200 mg/kg DM; Dove \& Mayes, 1991) are an order of magnitude lower than those in cabbage and spinach (1000$2000 \mathrm{mg} / \mathrm{kg}$ DM; current experiment). Herbage and faecal samples were analysed for $n$-alkanes using the method of Mayes et al. (1986).

\section{Statistical analysis}

The experiment was analysed using ANOVA with a factorial treatment structure (two treatments $\times$ four periods). Variance associated with the adaptation treatment was estimated between animals. Variance associated with period and interactions with period was estimated within animals. Rates of degradation of oxalic acid were expressed as means of values measured before and after the spinachcabbage feeding periods and were log transformed to reduce systematic variation of residuals. Data relating to the proportion of spinach in the diet were subjected to an arcsine transformation before statistical analysis, again, to reduce systematic variation of the residuals. Data were analysed using GENSTAT 5 (Lawes Agricultural Trust, 1989).

\section{Results}

Daily oral administration of oxalic acid had a marked influence on rates of degradation of oxalic acid in the rumen. Mean untransformed rates of degradation were 0.592 (range $0.236-1.685$ ) $\mu \mathrm{mol} / \mathrm{ml}$ rumen fluid per $\mathrm{h}$ for adapted animals and 0.269 (range $0.165-0.360) \mu \mathrm{mol} / \mathrm{ml}$ rumen fluid per $\mathrm{h}$ for control animals $(P<0 \cdot 05)$. Rates of degradation were not significantly affected by period and there was no significant interaction between period and treatment.

Concentrations of oxalic acid in spinach (5.85 (SE 0.76) $\mathrm{g} / \mathrm{kg} \mathrm{DM}$ ) were considerably greater than in cabbage which contained negligible amounts (0.17 (SE 0.05) g/kg DM). Cabbage had a higher nutritive value than spinach with lower levels of neutral-detergent fibre (213.5 (SE 12.80) $v$. 371.5 (SE 12.49) $\mathrm{g} / \mathrm{kg} \mathrm{DM}$ ) and acid-detergent fibre (199.2 (SE 17.60) v. 272.0 (SE 10.84) g/kg DM) and higher crude protein concentrations (134.7 (SE 12.07) v. 103.1 (SE 6.13) $\mathrm{g} / \mathrm{kg} \mathrm{DM}$ ). Average standing biomass was similar for the two plant species (1.91 (SE 0.62) v. 1.77 (SE 0.51) tonnes $\mathrm{DM} / \mathrm{ha}$ for cabbage and spinach respectively).

Total daily DM intakes from spinach and cabbage increased progressively over the four experimental periods (Fig. 1; $P<0.001$ ) averaging $0.20($ SE 0.034$) \mathrm{kg} \mathrm{DM} / \mathrm{d}$ during period 1 and reaching 1.20 (SE 0.115) kg DM/d during period 4 . The adaptation treatment had a small influence on DM intake with control animals tending to consume more than adapted animals (Fig. 1; 0.846 (SE 0.0996) v. 0.627 (SE 0.0878) kg DM/d; $P<0.1$ ).

The proportion of the spinach in the diet was significantly higher among oxalic-acid-adapted animals than among control animals (Fig. 2; 0.445 (SE 0.0293) v. 0.283 (SE 0.0177); $P<0.01$ ) over the whole experiment. The overall proportion of spinach in the diet decreased as the experiment progressed (0.462 (SE 0.0397), 0.357 (SE 0.0393), 0.314 (SE 0.0385 ), 0.292 (SE 0.0236) for periods $1-4 ; P<0.001$ ) but the interaction between adaptation treatment and period was not significant.

The intake of DM from spinach was not affected by adaptation treatment (Fig. 3) and intake of spinach increased over the course of the experiment (0.086 (SE 0.0109), 0.204 (SE 0.0209), 0.298 (SE 0.0412), 0.347 (SE 0.0431) kg DM/d for periods $1-4 ; P<0.001$ ). By contrast the intake of DM from cabbage was significantly lower among oxalic-acidadapted animals than among control animals (Fig. 4; $0 \cdot 372$ (SE 0.0264) v. 0.631 (SE 0.0783) kg DM/d; $P<0.05$ ). DM intakes from cabbage also steadily increased over the course of the experiment (0.116 (SE 0.0248), 0.399 (SE 0.0577), 0.685 (SE 0.0766), 0.852 (SE 0.0885) kg DM/d; $P<0.001)$.

\section{Discussion}

The experiment reported here was set up to test a hypothesis originally proposed by Provenza et al. (1992), that increased rates of rumen degradation of particular secondary plant compounds may result in greater selection by ruminant herbivores of plants containing those compounds. The hypothesis has not previously been tested in a free-grazing situation with choices of naturally occurring toxic plants. In 


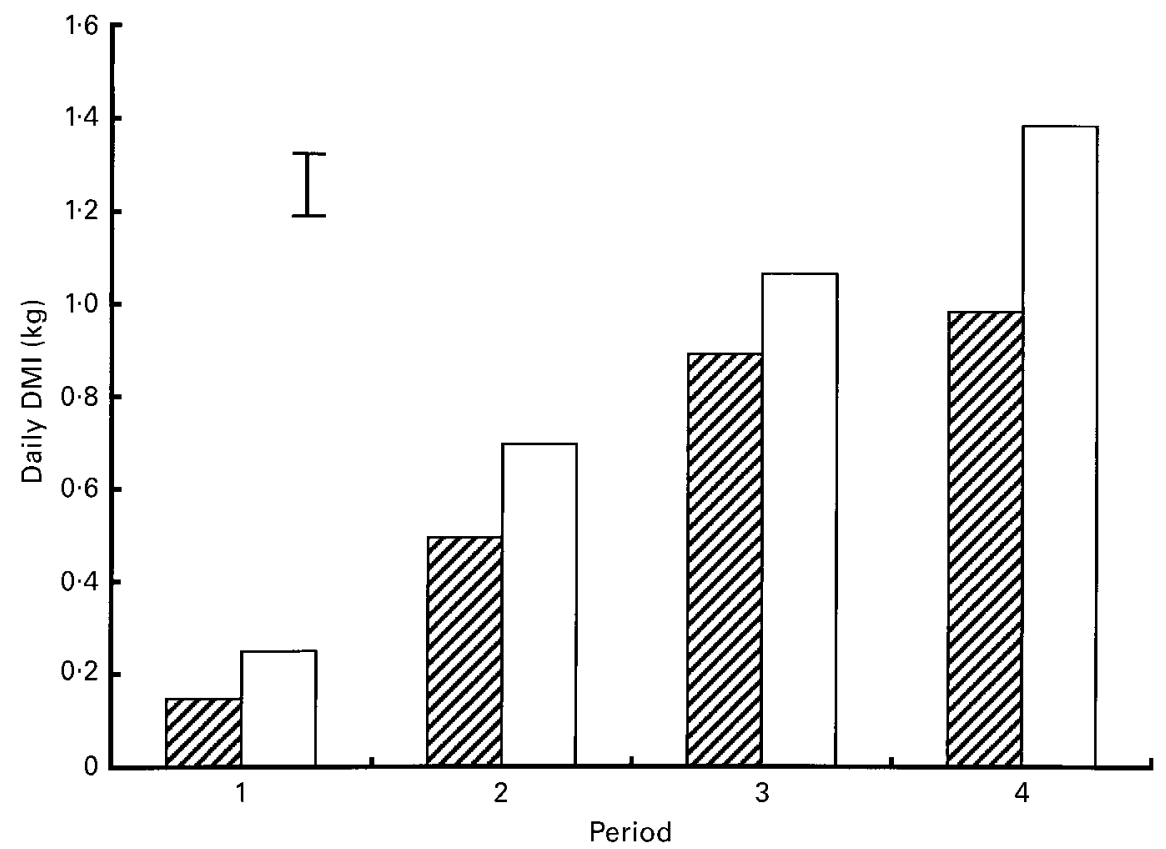

Fig. 1. Total daily dry matter intake (DMl, kg) of control goats ( $\square$; daily oral dosing with empty gelatin capsules) and goats adapted to oxalic acid (䐚; daily oral dosing with $0.6 \mathrm{mmol} / \mathrm{kg}$ live weight) offered a matrix of cabbage and spinach plants for $7 \mathrm{~h} / \mathrm{d}$ on two consecutive days per week during four consecutive 1-week periods. Values are means for six goats per treatment. The vertical bar represents the standard error of difference for the interaction between treatment and period.

an indoor experiment reported by Frutos et al. (1998), diet selection between two artificial feeds, differing only in their flavour and oxalic acid content, was not influenced by adaptation of the rumen to oxalic acid. In that experiment, however, oxalic acid concentrations in the diet did not present a serious toxic challenge to the animal; furthermore such effects may be more apparent in free-grazing situations where herbivores are making choices in circumstances more

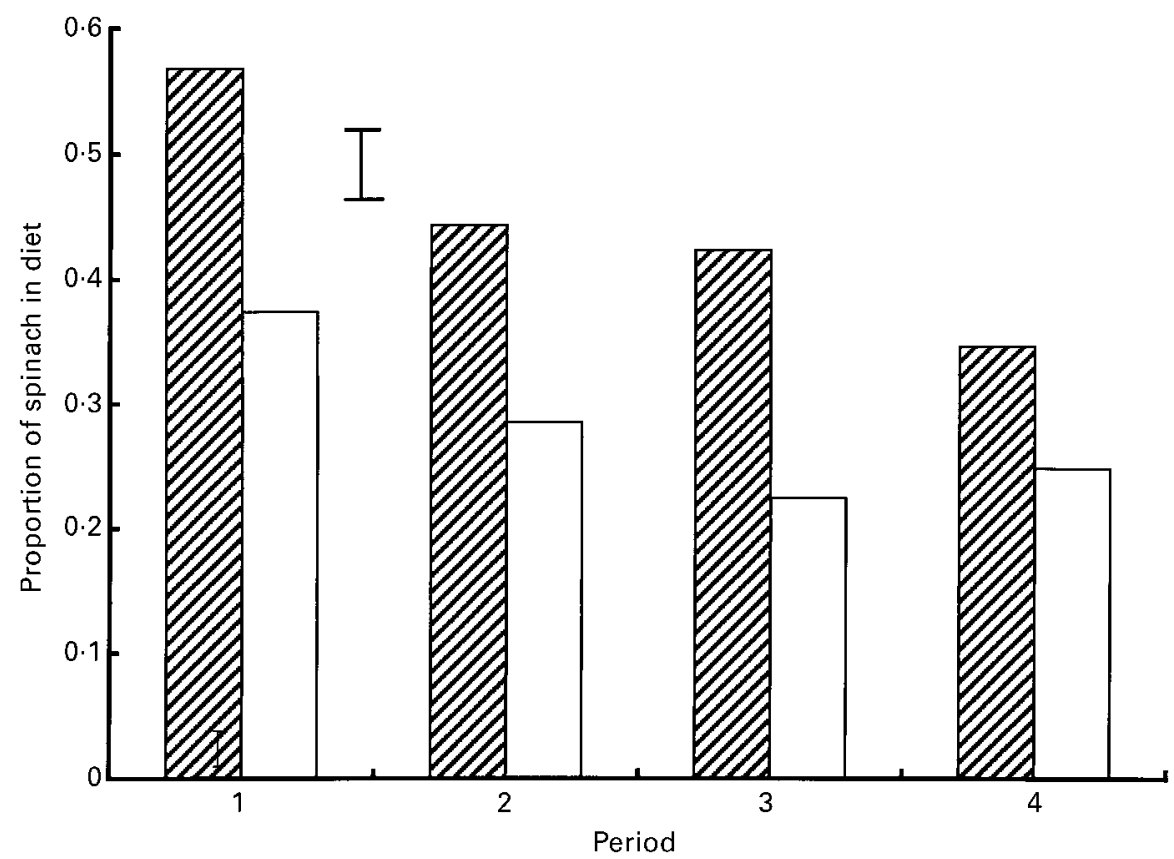

Fig. 2. Proportion of spinach in the diet selected by control goats ( $\square$; daily oral dosing with empty gelatin capsules) and goats adapted to oxalic acid (ש्थ; ; daily oral dosing with $0.6 \mathrm{mmol} / \mathrm{kg}$ live weight) offered a matrix of cabbage and spinach plants for $7 \mathrm{~h} / \mathrm{d}$ on two consecutive days per week during four consecutive 1-week periods. Values are means for six goats per treatment. The vertical bar represents the standard error of difference for the interaction between treatment and period (untransformed data). 


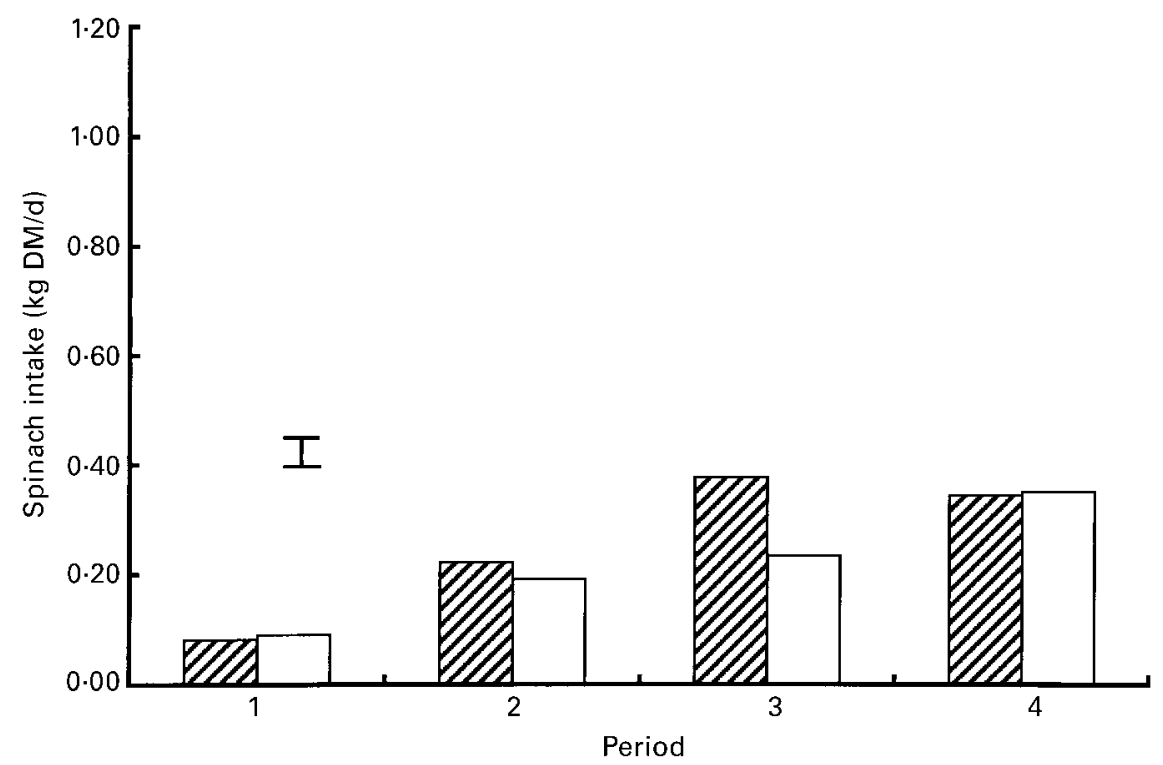

Fig. 3. Intake of dry matter from spinach $(\mathrm{kg} / \mathrm{d})$ by control goats ( $\square$; daily oral dosing with empty gelatin capsules) and goats adapted to oxalic acid (级; daily oral dosing with $0.6 \mathrm{mmol} / \mathrm{kg}$ live weight) offered a matrix of cabbage and spinach plants for $7 \mathrm{~h} / \mathrm{d}$ on two consecutive days per week during four consecutive 1-week periods. Values are means for six goats per treatment. The vertical bar represents the standard error of difference for the interaction between treatment and period.

akin to the environment in which their selective behaviour evolved.

In the current experiment, goats whose rumen microbial population was pre-adapted to oxalic acid showed higher rates of oxalic acid degradation in the rumen; absorption of dietary oxalic acid following consumption of spinach is therefore likely to have been less than in naïve animals.
Adapted animals selected a higher proportion, although not a higher amount, of spinach in their diets than did control animals. Control animals consumed greater proportions and greater amounts of cabbage than oxalic-acid-adapted animals. Thus, diet selection was modified by the oxalic acid treatment, but not in a manner consistent with the original hypothesis.

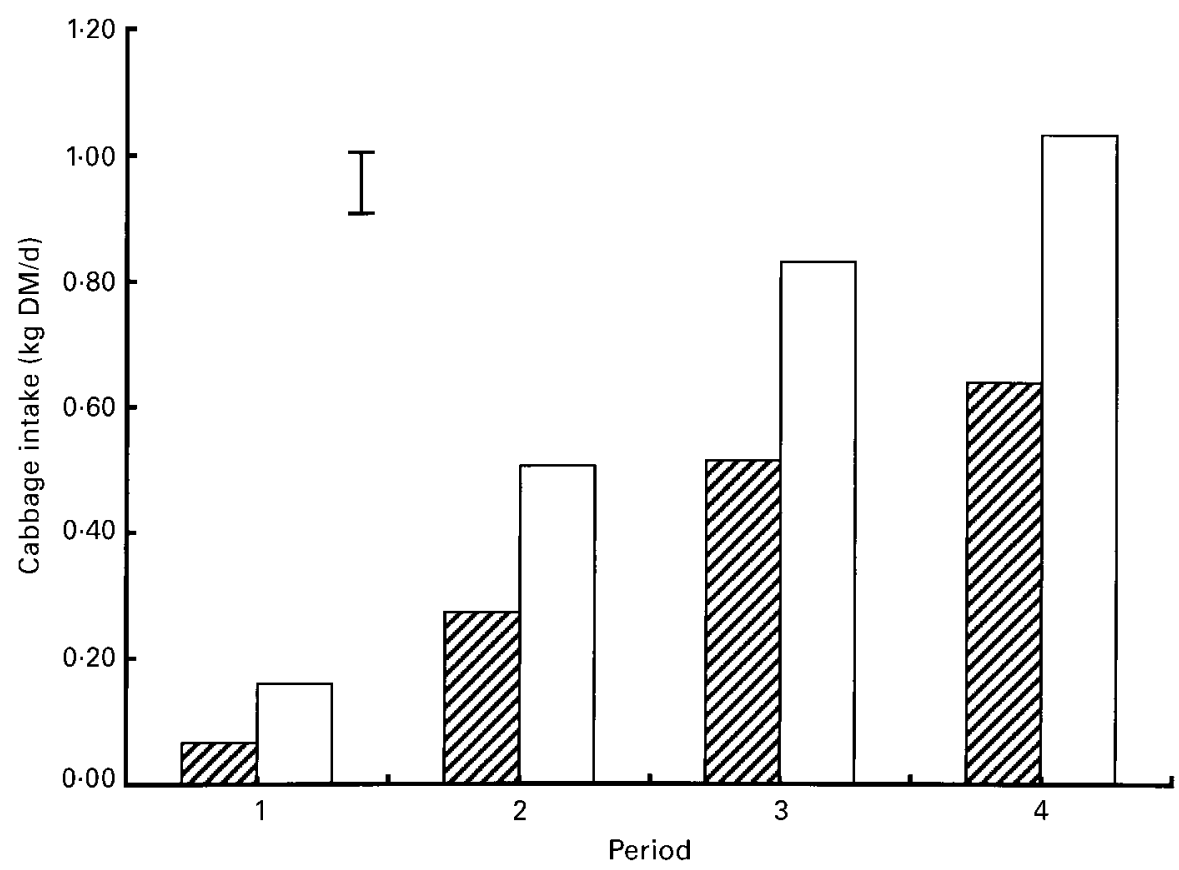

Fig. 4. Intake of dry matter from cabbage ( $\mathrm{kg} / \mathrm{d}$ ) by control goats ( $\square$; daily oral dosing with empty gelatin capsules) and goats adapted to oxalic acid (ש्Z; daily oral dosing with $0.6 \mathrm{mmol} / \mathrm{kg}$ live weight) offered a matrix of cabbage and spinach plants for $7 \mathrm{~h} / \mathrm{d}$ on two consecutive days per week during four consecutive 1-week periods. Values are means for six goats per treatment. The vertical bar represents the standard error of difference for the interaction between treatment and period. 
Differences in diet selection between adapted and control animals are unlikely to have been affected by food availability since the same relative proportions of the two diet options would have been available to all animals at any given point in time. Intakes of both diet options increased as the experiment progressed. This was probably due, in part, to the increasing familiarity of the experimental animals with the foods on offer. Initial caution with novel foods has been demonstrated before and probably represents an adaptive mechanism for avoiding over-consumption of potentially toxic plants (Provenza et al. 1994). Food neophobia also facilitates physiological adaptation to secondary compounds allowing time for induction of detoxification mechanisms at both rumen and systemic levels.

The higher voluntary intake of cabbage observed among control animals and the tendency of control animals to have higher total voluntary food intakes were unexpected. It may be that the adaptation dose of oxalic acid caused a general depression of rumen microbial activity or that it was partially absorbed and caused low-level systemic toxicity. This could explain the tendency for oxalic-acid-adapted animals to consume slightly less than control animals. The effect of the oxalic acid adaptation treatment on diet selection may have been mediated through effects on ruminal breakdown of other secondary compounds in the diet. The chosen 'non-toxic' food option was cabbage which itself contains secondary plant compounds such as glucosinolates and $S$-methyl cysteine sulfoxide. $S$-methyl cysteine sulfoxide is known to break down in the rumen to yield the haemolytic agent dimethyl disulfide which is responsible for the well-known haemolytic anaemia which results from chronic brassica consumption (Smith et al. 1974). In the current experiment, the short period of exposure $(2 \mathrm{~d}$ at a time) makes it highly unlikely that this route of toxicity could have influenced diet selection patterns. Glucosinolates are thought to break down in the rumen under the action of a plant thioglucosidase enzyme to yield a range of potentially toxic metabolites including isothiocyanates and nitriles (Duncan, 1991). Although these metabolites are potentially highly toxic, previous experiments have demonstrated that adaptation of rumen microbes can protect the host against toxicity (Duncan \& Milne, 1992). Chronic administration of oxalic acid, while increasing the rumen population of oxalicacid-degrading micro-organisms, may have had a negative impact on microbial breakdown of other secondary compounds such as the glucosinolate breakdown products through its effects on the rumen microbial population. The effect of this would be to increase the negative impact of glucosinolates among oxalic-acid-adapted animals. This is a possible explanation for the higher intake of cabbage among control animals.

These results raise interesting questions concerning the interaction between different groups of secondary compounds in the diet with respect to their toxic mechanisms and detoxification pathways. Such effects were unforeseen in the current experiment and in the absence of data collected to address these questions we cannot draw definitive conclusions about the role of glucosinolates and their metabolites in the observed patterns of diet selection. Interactions between secondary compounds have been demonstrated in other contexts (Freeland et al. 1985) and the effect of such interactions on diet choice has recently been examined in a series of preliminary indoor experiments (F.D. Provenza, personal communication). These experiments suggest that in some circumstances herbivores may select mixed diets to dilute the effects of individual secondary compounds but that in other situations toxins may interact to produce unexpected effects as found here. There is a need for closer examination of the role of interactions between the toxic mechanisms of different plant secondary compounds and the potential repercussions for diet selection behaviour.

Different diet selection patterns were observed in control and adapted animals despite the fact that animals grazed as one group. Social facilitation between animals has been found to influence diet selection patterns (Ralphs et al. 1994). This effect would have tended to dilute any treatment-related differences. Allowing treatment and control groups to graze separately might have led to greater differences in food selection patterns between control and adapted animals but would have introduced plot differences as a confounding factor. Although social facilitation can cause rapid extinction of conditioned food aversions in the absence of toxicosis, it is important to note that toxicosis can be as powerful as social facilitation in its effects on diet selection. For example, lambs do not continue to eat the food their mothers consume when the food continues to cause toxicosis (Provenza et al. 1993).

The long-term importance of rumen adaptation to plant secondary compounds as a factor influencing diet selection is likely to depend on the speed of induction of detoxification pathways. The rumen microbial population can respond rapidly to dietary changes and adaptation to oxalic acid can occur in a matter of a few days (Duncan et al. 1997). In the case of oxalic acid and other compounds to which the rumen adapts rapidly, long-term inter-animal differences in diet selection patterns are therefore unlikely to be related to differing rumen microbial states. For plant secondary compounds requiring longer intervals for adaptation to occur, the state of rumen adaptation could have a significant influence on diet selection patterns. Species differences in detoxification pathways (Duncan et al. 1997) may be partially responsible for the diet selection patterns found in different herbivore species.

In conclusion, this experiment demonstrates that adaptation to secondary compounds can modify diet selection patterns. The unexpected pattern of diet selection observed highlights the complexity of secondary compound biotransformation in the rumen but indicates that such chemical processes can have important consequences for free-grazing animals.

\section{Acknowledgements}

Pilar Frutos is grateful to the Spanish Council for Scientific Research (Consejo Superior de Investigaciones Científicas, CSIC, Spain) for the provision of a postdoctoral grant. Thanks are due to the many colleagues at MLURI who assisted in planting out the experimental plots and to Luc Chaverot for invaluable help with weeding. Pat Moberly conducted the proximate analyses of the herbage. A. M. Sibbald, I. J. Gordon and J. A. Milne made useful comments 
on draft manuscripts. The work was funded by the Scottish Office Agriculture, Environment and Fisheries Department.

\section{References}

Allison MJ, Dawson KA, Mayberry WR \& Foss JG (1985) Oxalobacter formigenes gen. nov., sp. nov.: oxalate-degrading anaerobes that inhabit the gastrointestinal tract. Archives of Microbiology 141, 1-7.

Allison MJ, Littledike ET \& James LF (1977) Changes in ruminal oxalate degradation rates associated with adaptation to oxalate ingestion. Journal of Animal Science 45, 1173-1179.

Dove H \& Mayes RW (1991) The use of plant wax alkanes as marker substances in studies of the nutrition of herbivores - a review. Australian Journal of Agricultural Research 42, 913-952.

Duncan AJ (1991) Glucosinolates. In Toxic Substances in Crop Plants, pp. 126-147 [JPF D’Mello, CM Duffus and JH Duffus, editors]. Cambridge: Royal Society of Chemistry.

Duncan AJ, Frutos P \& Young SA (1997) Rates of oxalic acid degradation in the rumen of sheep and goats in response to different levels of oxalic acid administration. Animal Science $\mathbf{6 5}$, 451-456.

Duncan AJ, Mayes RW, Lamb CS, Young SA \& Castillo I (1999) The use of naturally occurring and artificially applied n-alkanes as markers for estimation of short-term diet composition and intake in sheep. Journal of Agricultural Science 132, 233-246.

Duncan AJ \& Milne JA (1992) Rumen microbial degradation of allyl cyanide as a possible explanation for the tolerance of sheep to brassica-derived glucosinolates. Journal of the Science of Food and Agriculture 58, 15-19.

du Toit JT, Provenza FD \& Nastis A (1991) Conditioned taste aversions: how sick must a ruminant get before it learns about toxicity in foods. Applied Animal Behaviour Science 30, 35-46.

Freeland WJ, Calcott PH \& Anderson LR (1985) Tannins and saponin: interaction in herbivore diets. Biochemical Systematics and Ecology 13, 189-193.

Frutos P, Duncan AJ, Kyriazakis I \& Gordon IJ (1998) Learned aversion towards oxalic acid-containing foods by goats: does rumen adaptation to oxalic acid influence diet choice? Journal of Chemical Ecology 24, 383-397.

James LF (1972) Oxalate toxicosis. Clinical Toxicology 5, 231243.
Jefferies RL, Klein DR \& Shaver GR (1994) Vertebrate herbivores and northern plant communities: reciprocal influences and responses. Oikos 71, 193-206.

Lawes Agricultural Trust (1989) Genstat 5. Oxford: Oxford University Press.

Libert B (1981) Rapid determination of oxalic acid by reversedphase high performance liquid chromatography. Journal of Chromatography 210, 540-543.

Mayes RW, Lamb CS \& Colgrove PM (1986) The use of dosed and herbage $n$-alkanes as markers for the determination of herbage intake. Journal of Agricultural Science 107, 161-170.

Palo RT \& Robbins CT (1991) Plant Defenses Against Mammalian Herbivory. Boca Raton, FL: CRC Press, Inc.

Provenza FD \& Balph DF (1987) Diet learning by domestic ruminants: theory, evidence and practical implications. Applied Animal Behaviour Science 18, 211-232.

Provenza FD, Lynch JJ, Burritt EA \& Scott CB (1994) How goats learn to distinguish between novel foods that differ in postingestive consequences. Journal of Chemical Ecology 20, 609-624.

Provenza FD, Lynch JJ \& Nolan JV (1993) The relative importance of mother and toxicosis in the selection of foods by lambs. Journal of Chemical Ecology 19, 313-323.

Provenza FD, Pfister JA \& Cheney CD (1992) Mechanisms of learning in diet selection with reference to phytotoxicosis in herbivores. Journal of Range Management 45, 36-45.

Ralphs MH, Graham D \& James LF (1994) Social facilitation influences cattle to graze locoweed. Journal of Range Management 47, 123-126.

Smith GS (1992) Toxification and detoxification of plant compounds by ruminants: an overview. Journal of Range Management 45, 25-30.

Smith RH, Earl CR \& Matheson NA (1974) The probable role of S-methyl cysteine sulphoxide in kale poisoning in ruminants. Transactions of the Biochemical Society 2, 101-104.

Van Soest PJ (1963) Use of detergents in the analysis of fibrous feeds II. A rapid method for the determination of fiber and lignin. Journal of the Association of Official Analytical Chemists 46, 829-835.

Van Soest PJ \& Wine RH (1967) Use of detergents in the analysis of fibrous feeds IV. Determination of plant cell-wall constituents. Journal of the Association of Official Analytical Chemists 50, 50-55. 